\title{
Circuit
}

\section{Introduction. Engagements sonores : éthique et politique}

\author{
Luis Velasco-Pufleau
}

Engagements sonores

Volume 28, numéro 3, 2018

URI : id.erudit.org/iderudit/1055190ar

https://doi.org/10.7202/1055190ar

Aller au sommaire du numéro

Éditeur(s)

Les Presses de l'Université de Montréal

ISSN 1183-1693 (imprimé)

1488-9692 (numérique)

Découvrir la revue

Citer cet article

Velasco-Pufleau, L. (2018). Introduction. Engagements sonores :

éthique et politique. Circuit, 28(3), 9-12. https://

doi.org/10.7202/1055190ar Ce document est protégé par la loi sur le droit d'auteur. L'utilisation des services d'Érudit (y
Tous droits réservés $\odot$ Circuit, musiques contemporaines, 2018 compris la reproduction) est assujettie à sa politique d'utilisation que vous pouvez consulter en
ligne. [https://apropos.erudit.org/fr/usagers/politique-dutilisation/] 


\section{Introduction. Engagements sonores: éthique et politique}

Luis Velasco-Pufleau

"If ethics without politics is empty, then politics without ethics is blind" ", écrit le philosophe Simon Critchley. Selon lui, au cœur d'une politique émancipatrice se trouve un moment éthique - qu'il qualifie de métapolitique - qui apporte au sujet «the motivational force or propulsion into political action ${ }^{2} »$. Toutefois, bien qu'éthique et politique ne soient pas des domaines hétérogènes, leur mise en relation ne va pas de soi. L'analyse de cette relation doit s'appuyer sur des situations et des processus singuliers. Ce numéro de Circuit se propose d'explorer les liens entre éthique et politique dans la création musicale des $\mathrm{Xx}^{\mathrm{e}}$ et $\mathrm{XxI}^{\mathrm{e}}$ siècles. Son objectif est d'examiner la dimension politique des situations dans lesquelles l'action musicale a lieu ainsi que la façon dont les compositeur.rice.s, les musicien-ne.s et les auditeur.rice.s mobilisent une réflexion éthique au sein d'événements qui les obligent à penser d'une façon nouvelle leur rapport au monde.

Il s'agit donc d'un double questionnement. D’une part, ce numéro interroge le rapport entre la musique - les œuvres et les pratiques musicales - et la politique, plus particulièrement ce que Jacques Rancière appelle le «partage du sensible ${ }^{3}$ » la distribution des places et des identités, le découpage des espaces et des temps, de ce qui est visible et de ce qui ne l'est pas, de ce qui est audible, du bruit, de la parole et du silence. En pensant la politique audelà de l'administration des fonctions régaliennes de l'État (forces armées, justice, police), de l'exercice du pouvoir, de l'ordre social et du consensus, le rapport entre musique et politique se dévoile dans la façon dont les œuvres et les pratiques musicales «contribuent à dessiner un paysage nouveau du visible, du dicible et du faisable ${ }^{4}$ ». Le moment de cette reconfiguration du partage du sensible est le «dissensus». La contribution des œuvres et des pratiques musicales à ce moment de basculement réside dans leur capacité à
1. Simon Critchley (2007), Infinitely Demanding: Ethics of Commitment, Politics of Resistance, Londres, New York, Verso, p. 13.

2. Ibid.

3. Jacques Rancière (2004), "L'esthétique comme politique", in Malaise dans l'esthétique, Paris, Galilée, p. 38.

4. Jacques Rancière (2008), "Les paradoxes de l'art politique", in Le spectateur émancipé, Paris, La fabrique, p. 84. 
5. Ibid., p. 66.

6. Pour une critique de cette dérive, voir "Le tournant éthique de l'esthétique et de la politique", in Rancière (2004), op. cit., p. 143-173.

7. Alain Badiou (2003), L'Éthique: essai sur la conscience du mal, Caen, Nous, p. 37. Pour l'auteur, au lieu de lier l'éthique "à des catégories abstraites (I'Homme, le Droit, l'Autre...), on le rapportera à des situations" (p. 21). De ce fait, "il n'y a pas d'éthique en général. II n’y a - éventuellement qu'éthique de processus par lesquels on traite les possibles d'une situation" (p. 38).

8. Ibid., p. 58.

9. Ibid., p. 99 .

10. Hannah Arendt (2014), Qu'est-ce que la politique?, Paris, Seuil, p. 289. provoquer des ruptures dans l'ordre politique «par l'invention d'une instance d'énonciation collective qui redessine l'espace des choses communes ${ }^{5}$ ».

D'autre part, il s'agit d'étudier comment la création musicale s'inscrit au sein de processus singuliers qui font appel à une réflexion éthique qui a pour but la recherche du bien commun et de la justice sociale. Cela n’a rien à voir avec une volonté de soumettre les œuvres et les pratiques musicales à une évaluation utilitariste ou à un jugement moral ${ }^{6}$. Ces processus montrent plutôt des façons particulières de s'engager dans une situation, de lui être fidèle en traitant «le possible de cette situation jusqu'au bout », c'est-à-dire de faire «advenir, dans la mesure du possible, ce que cette situation contient d'humanité affirmative" ». Ainsi, d'après Alain Badiou, le but de l'engagement éthique est de contribuer à faire advenir des "possibles encore inexplorés ${ }^{8}$ ».

Au cœur du rapport entre création musicale, éthique et politique se trouve la réflexion sur les moyens et les possibilités propres à l'écoute et à la musique, et plus largement au sonore, d'agir sur le réel. D’une façon générale, cette réflexion s'appuie et se développe en lien avec un événement qui «fait advenir "autre chose" que la situation, que les opinions, que les savoirs institués ${ }^{9}$ ». L'article de Laurent Feneyrou, qui ouvre le Dossier thématique de ce numéro, explore l'opéra La Petite Fille aux allumettes (1997) de Helmut Lachenmann en rapport avec l'éruption de la violence terroriste en Allemagne de l'Ouest dans les années 1960-1970. Il montre de quelle façon les actions de la Fraction armée rouge (Rote Armee Fraktion, RAF) s'inscrivent au sein d'une situation qui oblige le compositeur à repenser de façon politique son travail avec les sons. Il répond par des gestes nouveaux qui refusent les conventions, par de nouvelles modalités d'écoute qui bouleversent les cadres consensuels. Impossible pour Lachenmann de ne pas prendre position, avec les moyens propres à son métier de compositeur, à l'intérieur de cette nouvelle configuration de la violence.

Les guerres sont des événements qui ont bouleversé durablement l'ordre politique aux $\mathrm{Xx}^{\mathrm{e}}$ et $\mathrm{XXI}^{\mathrm{e}}$ siècles. Ainsi que l'affirmait déjà Hannah Arendt pour le $\mathrm{Xx}^{\mathrm{e}}$ siècle, «ce sont les guerres et les révolutions [...] qui constituent les expériences politiques fondamentales de notre siècle. Les ignorer reviendrait à ne jamais avoir vécu dans le monde qui est le nôtre ${ }^{10}{ }$. L'article de José Luis Besada et d'Ainara Zubizarreta explore les résonances du bombardement de la ville de Guernica - qui eut lieu le 26 avril 1937, durant la Guerre civile espagnole - dans les œuvres de trois compositeurs basques, écrites entre 1966 et 2013 . Les auteur.e.s étudient la fonction symbolique de ce crime de guerre au sein de ces œuvres et montrent de quelle façon la dimension politique de la musique émerge à l'intérieur du cadre conflictuel 
délimité par la mémoire de la Guerre civile espagnole, le pouvoir autoritaire de la dictature franquiste et la violence terroriste menée au Pays basque par Euskadi Ta Askatasuna (Pays basque et liberté, ETA).

Les modalités des débats sur les liens entre création musicale et politique délimitent également la réception des œuvres et des pratiques musicales. Pendant la période des révoltes internationales de 1968, le festival Automne de Varsovie [Warszawska Jesień] constituait un lieu privilégié d'échange entre des compositeurs dont les activités évoluaient au sein de régimes politiques radicalement différents. L'article de Monika Żyła étudie la réception de la musique du compositeur britannique Cornelius Cardew à ce festival durant les années 1960 et au début des années 1970. Lauteure explore le lien entre l'interprétation des idées politiques à la base de la création musicale de Cardew et les débats qui ont eu lieu suivant l'écoute de ses œuvres à Varsovie. Żyła montre de quelle façon le partage du sensible structure aussi bien les rapports de pouvoir au sein du rituel du concert que les opinions sur les conditions d'inscription politique de l'écoute musicale.

En effet, l'écoute peut être considérée comme une activité délibérée qui constitue le sujet en rapport avec son milieu sonore. En tant que processus, elle se situe au cœur des rapports politiques entre les individus. L'article de Makis Solomos explore de quelle façon l'écoute musicale peut être appréhendée comme construction du commun. Prenant comme point de départ la théorie des communs et l'écologie acoustique, notamment le travail de la compositrice canadienne Hildegard Westerkamp, sa réflexion porte sur les moyens de repolitiser la musique à partir de son inscription dans l'espace commun. L'écoute commune en tant qu'expérience politique est aussi au cœur de l'Exclusivité web du numéro. Il s'agit d'un recueil exceptionnel de données sonores liées au mouvement social du printemps québécois de 2012, réalisé par Emanuelle Majeau-Bettez et Symon Henry. Présenté sous la forme d'un carnet de recherche en accès ouvert sur la plateforme Hypothèses ${ }^{11}$, le recueil «Québec : traces sonores du printemps 2012» permet aux lecteur.rice-s d'explorer l'atmosphère toute particulière du mouvement social du printemps québécois.

L'exploration critique de l'histoire récente du Québec est aussi mise à l'honneur dans l'entretien que j'ai réalisé avec le compositeur québécois René Lussier à propos de son œuvre Le trésor de la langue (1989). Lussier y raconte la réception de son œuvre et nous parle de son processus de création, de ses motivations et de sa conception d'une éthique du son. En rapprochant des matériaux hétérogènes grâce à la technique du collage sonore, Lussier a créé une œuvre politique qui fait entendre la voix des personnes ordinaires, juxta-
11. Voir https://quebec2012. hypotheses.org (consulté le 16 octobre 2018). 
12. Hannah Arendt (2005), Journal de pensée, Paris, Seuil, p. 28 posée à un discours de Charles de Gaulle ou au manifeste du FLQ. Le Cahier d'analyse réalisé par Ana Dall'Ara-Majek sur Le trésor de la langue développe remarquablement les propos du compositeur.

Enfin, l'artiste Silvana Gallinotti a réalisé les illustrations de ce numéro en prenant comme point de départ la pensée politique de Hannah Arendt. Par son travail sur le corps humain, et tout particulièrement le corps féminin dans sa série «Paysages humains», Gallinotti explore l’idée selon laquelle «la politique repose sur un fait: la pluralité humaine ${ }^{12} »$.

Bonne lecture!

Fribourg, septembre 2018 .

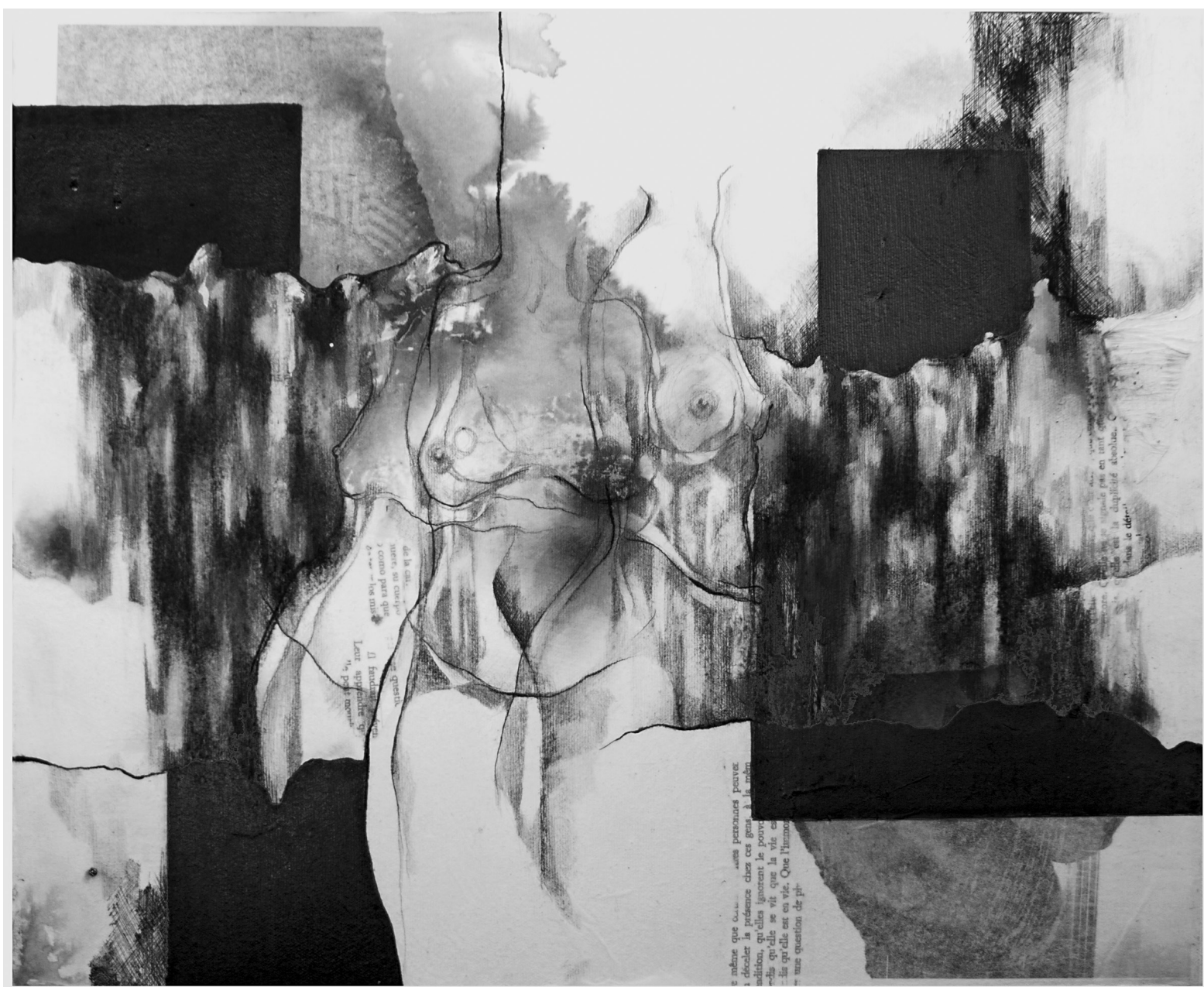

